\title{
Satisfacción del profesorado y alumnado con el empleo de Metodologías de Aprendizaje Colaborativo mediada por las TIC: Dos estudios de casos
}

\author{
Satisfaction of teachers and students with the use of Collaborative Learning \\ Methodologies mediated by ICT: Two case studies
}

\author{
Rosalba Acosta Corporan ${ }^{a}$, Azucena Hernández Martín ${ }^{b}$ \\ Antonio Víctor Martín García \\ ${ }^{a}$ Facultad de Educación, Universidad de Salamanca, España. \\ u139132@usal.es \\ ${ }^{b}$ Departamento de Didáctica y Organización Escolar y MIDE, Universidad de Salamanca, España. \\ azuher@usal.es \\ ${ }^{c}$ Departamento de Teoría e Historia de la Educación, Universidad de Salamanca, España. \\ avmg@usal.es
}

\section{RESUMEN}

En este artículo se evalúa el nivel de satisfacción del profesorado y alumnado cuando trabajan con metodologías de aprendizaje colaborativo a través de las Tecnologías de la Información y la Comunicación en el proceso de enseñanza-aprendizaje. Para tal fin se analizaron varios estudios de casos en donde se exploraron las fortalezas y debilidades con que se encuentran los alumnos y profesores cuando emplean estas experiencias. El estudio se realizó en República Dominicana (nivel Secundario o Bachillerato) y se desarrolló con una muestra de 57 alumnos y 2 docentes. Se empleó el método mixto para analizar las fichas pedagógicas utilizadas por docentes y observadores y, la rúbrica cumplimentada por el alumnado. Los hallazgos ponen de manifiesto que la evaluación de las actividades en contextos de aprendizaje colaborativo con TIC sigue siendo un punto débil para el docente, quien tiene miedo de equivocarse y valorar erróneamente el trabajo de los estudiantes.

Palabras claves: Aprendizaje colaborativo, Rol docente, Aprendizaje basado en problemas, Competencias Digitales.

\section{ABSTRACT}

This article evaluates the level of satisfaction of teachers and students when they work with collaborative learning methodologies through Information and Communication Technologies in the teaching-learning process. For this purpose, several case studies were analyzed where the strengths and weaknesses that students and teachers encounter when they use these experiences were explored. The study it has been made in the Dominican Republic (secondary or high school) and was carried out with a sample of 57 students and 2 teachers. The mixed method was used to analyze the pedagogical records used by teachers and observers and the rubric completed by the students. The findings show that the evaluation of activities in contexts of collaborative learning with ICT remains a weak point for the teacher, who is afraid of making mistakes and misjudging the students work.

Key words: Collaborative Learning, Teaching role, Problem-based learning, Digital competences. 
Estudios Pedagógicos XLVII N² 2: 79-97, 2021

SATISFACCIÓN DEL PROFESORADO Y ALUMNADO CON EL EMPLEO DE METODOLOGÍAS DE APRENDIZAJE COLABORATIVO MEDIADA POR LAS TIC: DOS ESTUDIOS DE CASOS

\section{INTRODUCCIÓN}

La evolución constante de las Tecnologías de la Información y la Comunicación (TIC en lo sucesivo) generan nuevos escenarios de aprendizaje y obligan a la escuela a replantearse los métodos de enseñanza tradicionales insuficientes ya para responder a las exigencias de un mundo globalizado. Este cambio conlleva a la reflexión, tanto de las autoridades educativas como de los docentes, quienes además de desarrollar los contenidos curriculares, deben hacer frente a nuevas competencias y aprendizajes significativos adaptados a la sociedad actual. Este argumento, es defendido por grandes organizaciones como la Organización para la Cooperación y el Desarrollo Económicos (OCDE), la Organización de las Naciones Unidas para la Educación, la Ciencia y la Cultura (UNESCO), Organización de Naciones Unidas (ONU), entre otras, las cuales apuestan firmemente por un modelo global educativo que corrobore con la formación de un alumnado en competencias claves para el siglo XXI (Portillo-Torres, 2017).

Seles denomina competencias claves por considerarse como requisitos imprescindibles para funcionar eficazmente en esta sociedad. En diversas fuentes se identifican dichas competencias y aptitudes, (Almerich et al., 2019; Assiter, 2017; Schwanitz, 2018; Vaillant y Zidán, 2018; Van-Laar et al., 2017). Algunas de las más destacadas son: pensamiento crítico, resolución de problemas, capacidad de liderazgo y colaboración, iniciativa, comunicación oral y escrita eficaz, etc. No obstante, lamentablemente rara vez estas son tomadas en consideración en la práctica docente (Vaillant y Zidán, 2018).

Si bien es cierto, que para desarrollar este conjunto de competencias y actitudes se pueden adoptar distintos métodos de enseñanza. En ese sentido, Vega-Gil (2016), propone que siempre debe prevalecer aquel que se adapte mejor al contexto. Por ello, se han de tomar en cuenta los nuevos entornos colaborativos mediados por las TIC, los cuales ofrecen una gama de recursos que permiten implementar diversas estrategias y metodologías, orientadas a la formación de este tipo de competencias, tanto en los docentes como en los estudiantes. Estos ambientes de aprendizaje han dado origen a lo que la comunidad científica denomina CSCL (Computer Supported Collaborative Learning) Aprendizaje Colaborativo con Soporte del Computador. Cuando hablamos de CSCL nos referimos a un conjunto de actividades en las que dos o más estudiantes interactúan y se comprometen mutuamente para lograr uno o varios objetivos de aprendizaje con el soporte de las TIC (Gikandi, 2020; Johnson y Johnson, 2009; Koschman et al., 1996; Reis et al., 2017).

Lo que distingue esta metodología de otros enfoques de aprendizaje es su énfasis en la interacción social y el compromiso mutuo para alcanzar el éxito (Sangrà et al., 2012). De modo que, cuando se implican todos los participantes y asumen el rol que le corresponde existen mayores probabilidades de alcanzar los objetivos propuestos. Se trata entonces de aprender haciendo, pero con apoyo de los compañeros de equipo.

Otros beneficios que señala la literatura científica (Johnson y Johnson, 2009; Torrego y Negro, 2012; Tamargo y Rodríguez, 2015), están relacionados con el establecimiento de relaciones entre el alumnado mucho más positivas, caracterizadas por la simpatía, la atención, la generosidad y el respeto mutuo, así como sentimientos recíprocos de responsabilidad y de ayuda mutua, aceptación de las diferencias entre los estudiantes y la modificación de las relaciones interpersonales en cantidad y calidad. Tales actitudes contagian a todos los actores educativos. 
Para que el CSCL sea eficaz ha de considerarse sus limitaciones, pero también sus inconvenientes. De hecho, se achaca que muchos de los fracasos y frustraciones se producen cuando se utilizan inadecuadamente los recursos en los entornos tecnológicos, cuando existe indisposición por parte de los actores, etc. Algunos de los casos más comunes se dan, por ejemplo, cuando no se fijan objetivos en común, el plan de clase no correlaciona con las herramientas, se utilizan los recursos TIC como un fin, no como un medio, la inexperiencia para navegar en el entorno TIC, pero sobre todo la "escasa formación del profesorado" para diseñar e implementar estas metodologías (Acosta et al., 2019; Acosta et al., 2020; GarcíaMerino et al., 2016; Hernández et al., 2014; Kumar et al., 2010).

Gran parte del cambio de estrategia en favor del uso de entornos de enseñanza apoyados en TIC se ha puesto de manifiesto en numerosos foros internacionales en los últimos años (Esteve, 2009; Lugo y Ithurburu, 2019; Unesco, 2015). El enfoque principal de estos lineamientos se centra en la reducción de la brecha digital (acceso a las Tecnologías de la Información y la Comunicación y manejo de las mismas) y, en menor medida, en una formación inicial y continua de calidad para el profesorado, que le permita utilizar metodologías vanguardistas como el CSCL y, por ende, mejorar sus prácticas.

Algunos países como Finlandia, Singapur, Dinamarca, Japón, entre otros han apostado claramente por un modelo educativo basado en la atracción, formación y retención de docentes y empleo de nuevas metodologías, tales como el aprendizaje colaborativo mediado por las TIC para la enseñanza. Estos lineamientos les han valido para ser referentes del sistema educativo a nivel mundial por los resultados satisfactorios obtenidos (Auld et al., 2019; Vega-Gil, 2016). Sin embargo, como ponen de manifiesto algunos trabajos (Acosta et al., 2019; Cañón et al., 2016), las infraestructuras tecnológicas no están llegando acompañadas de políticas apropiadas para mejorar la calidad educativa en otros muchos países. Es el caso de Latinoamérica, donde la mayor parte de sus países se encuentran muy por debajo de la media de la OCDE con respecto a el rendimiento y calidad de la enseñanza, estando en los últimos lugares Republica Dominicana (RD) (Vaillant y Zidán, 2018). Por ser objeto de nuestra investigación nos centraremos especialmente en este país.

En RD actualmente se trabaja en la reforma curricular de un modelo de más de 20 años de antigüedad basado en la transmisión de conocimientos y, se están incorporando nuevos paradigmas ligados a las Tecnologías de la Información y la Comunicación (TIC) para mejorar la calidad educativa. Entre estos se encuentran los PPA (Proyecto Participativo de Aula). Se trata de un enfoque que plantea dar solución a una problemática que afecta a la sociedad, centro educativo, comunidad, etc. Dicha perspectiva debe ser asumida por todos los agentes del centro educativo, quienes trabajan en colaboración para ejecutar el proyecto, adecuando el eje transversal a los contenidos específicos de cada asignatura. El modelo responde a la necesidad de impulsar una pedagogía crítica y transformadora; capaz de articular las experiencias de aula con la realidad social de los sujetos involucrados en los procesos de aprendizaje. (investigación-acción) (Imbernon y Medina, 2008; MINERD, 2017).

Desde esta perspectiva, es como se desarrollan los dos estudios de casos que se presentan en este trabajo. Estos fueron realizados en el Instituto Politécnico Fabio Amable Mota, de Santo Domingo, República Dominicana. Los docentes que participaron recibieron una formación previa en CSCL de unas 30 horas y diseñaron un proyecto para ponerlo en marcha en el curso escolar con la supervisión de evaluadores cualificados. La población observada fue de 2 docentes y 57 alumnos. 
Con el análisis de los casos se pretende evaluar el nivel de satisfacción del profesorado y alumnado cuando trabajan con las metodologías de aprendizaje colaborativo mediadas por las TIC en el proceso de enseñanza-aprendizaje. Así mismo, se pretendía comprobar si existía alguna correlación entre el rol docente y la satisfacción del alumnado según el grado o nivel educativo, identificar las fortalezas y debilidades con que se encuentran los alumnos y profesores cuando emplean estas experiencias, analizar las valoraciones del docente, alumnado y observador sobre los contenidos, metodologías, tiempo, agrupamiento, y proceso de evaluación del proyecto y, comparar las diferencias y similitudes en las opiniones aportadas. Además, se realiza un análisis factorial para establecer las variables de análisis.

\section{MÉTODO}

Tal como se ha indicado, esta investigación está basada en dos estudios de caso, llevados a cabo en Santo Domingo, República Dominicana, en el Politécnico de Educación Secundaria Fabio Amable Mota. El programa de formación de los docentes se inició en el año 2017 y posteriormente se realizó el trabajo de campo para la recogida de información y evaluación de resultados. En un primer momento se realizaron, cinco casos-proyectos de tipo colaborativo sobre varias temáticas. Finalmente se seleccionaron dos de estos proyectos por responder mejor a los objetivos del estudio.

La temática que se desarrolló en ambos proyectos fue "colaboremos con un ambiente sano y sostenible". Dicho tema fue elegido por los alumnos de los centros educativos, siguiendo el criterio del Proyecto Participativo de Aula (PPA). Para la selección del tema se propusieron varios tipos de problemas que afectaban al centro educativo y/o comunidad tales como el buying, el embarazo en la adolescencia, colaboremos por un ambiente sano y sostenible, entre otros. Siendo el más votado este último. Para el análisis de estos casos se optó por el método mixto (cualitativo y cuantitativo) debido a que, por un lado, se recogen datos cualitativos a través de la observación los cuales se plasman en fichas y, por otro, se analizan datos cuantitativos proporcionados por una rúbrica cumplimentada por el alumnado. Además, se realiza un análisis factorial a partir de las variables consideradas en dicho instrumento (Brannen, 2017).

\subsection{PARTICIPANTES}

El número final de participantes en los proyectos fue de 57 alumnos $(25$ eran de tercero de bachillerato, especialidad informática y 32 de cuarto de bachillerato, especialidad informática) y 2 profesores (del área de inglés). De estos, los observadores o evaluadores de los casos fueron: una directora de primaria (evaluadora caso A) y el sub-director del centro educativo (evaluador caso B).

\subsection{INSTRUMENTOS}

La recogida de datos se ha realizado mediante el empleo de diferentes instrumentos diseñados e implementados previa validación por el grupo de investigación GITE-USAL (Grupo de Investigación en Innovación y Tecnología Educativa de la Universidad de Salamanca). 
Estos habían sido empleados en un proyecto similar en Castilla y León, España (GarcíaValcárcel, 2015). Se trata de dos fichas y una rúbrica. En la tabla 1 se describen los aspectos que incluían los distintos instrumentos utilizados.

Tabla 1. Aspectos que incluyen los instrumentos analizados

\begin{tabular}{|c|c|l|}
\hline Destinatarios & Instrumentos & \multicolumn{1}{|c|}{ Objetivos } \\
\hline Profesorado & Ficha & $\begin{array}{l}\text {-Describir del proyecto } \\
\text {-Evaluar del proyecto en sus distintas fases (inicio, } \\
\text { desarrollo y final) } \\
\text {-Identificar el grado de satisfacción del profesorado } \\
\text { con relación al proyecto }\end{array}$ \\
\hline Observador & Ficha & $\begin{array}{l}\text {-Características del proyecto } \\
\text {-Seguimiento del proyecto } \\
\text {-Evaluación del proyecto (inicio, desarrollo y final) }\end{array}$ \\
\hline Alumnado & Rúbrica & $\begin{array}{l}\text {-Datos del proyecto } \\
\text {-Satisfacción con el proyecto }\end{array}$ \\
\hline
\end{tabular}

Fuente. Elaboración propia.

La primera guía o ficha estaba destinada para el profesor. A través de esta los docentes debían aportar una descripción general de su proyecto, pudiéndose cumplimentar con carácter semanal, quincenal o mensual, dependiendo del tiempo de duración de este. La ficha permitía contestar a los distintos aspectos del caso estudiado, de acuerdo con los siguientes criterios de valoración: algo, mucho y nada (ver tabla. 1).

La segunda ficha estaba dirigida al observador, quien a través tres visitas al aula en distintas fases del proyecto (inicio, desarrollo y final) iba evaluando la evolución del proyecto, recogiendo informaciones de forma descriptiva, abierta, cualitativa o cuantitativa si así se consideraba necesario, sin intervenir u obstaculizar la actividad cotidiana. Finalmente, la tercera estaba destinada al alumnado. La misma se cumplimentó una vez finalizado el proyecto y se concretó en un cuestionario sobre datos del centro, del alumnado y del proyecto y un diferencial semántico en el cual podían expresar su satisfacción o no con el proyecto realizado por su profesor. La rúbrica estaba distribuida en dos polos opuestos con escala de 1 a 7.

\subsection{PROCEDIMIENTO DE RECOGIDA DE DATOS Y ANÁLISIS DE LA INFORMACIÓN}

Los casos analizados tuvieron una duración de tres meses. Con respecto a los criterios de recogida de información se tomó en cuenta el siguiente protocolo.

1. Solicitar la participación de los docentes que así lo desearan al estudio de caso e invitarles a poner sus datos de contacto. 
Estudios Pedagógicos XLVII N² 2: 79-97, 2021

SATISFACCIÓN DEL PROFESORADO Y ALUMNADO CON EL EMPLEO DE METODOLOGÍAS DE APRENDIZAJE COLABORATIVO MEDIADA POR LAS TIC: DOS ESTUDIOS DE CASOS

2. Contactar con los docentes para conocer la fecha de inicio y fin de los proyectos para planificar las secciones de observación.

3. Enviar las fichas por e-mail a los docentes para que se familiarizaran con la misma. De esta forma, se pudo resolver las dudas sobre el material. De forma adicional se realizó una videoconferencia con todos los docentes participantes para aclarar cualquier otra incertidumbre.

4. Concertar o confirmar cita con el docente para realizar la observación y preguntarle si lo estaba realizando en colaboración con otros.

5. Se creó un chat en Facebook para abordar cuestiones relacionadas al proyecto. Por ejemplo, se compartían la planificación, evidencias, entre otras.

Para mostrar unos hallazgos de mejor calidad se procedió a realizar un análisis factorial de dicho instrumento con el programa estadístico Unrestricted Factor Analysis. Version 10.4.01 x64bits. 2016. Se trata de un software libre desarrollado por los profesores de la Universitat Rovira i Virgili Urbano Lorenzo-Seva y Pere J. Ferrando.

El análisis factorial implicó realizar un procedimiento de limpiado, buscando casos atípicos o encuestados no comprometidos con la investigación (d2 de Mahalanobis); se comprobó el rango intercuartílico, el grado de correlación de los ítems, el índice de adecuación muestral KMO (Kaiser-Meyer-Olkin), un análisis factorial exploratorio y el análisis paralelo para ver si procedía o no efectuar el análisis factorial. El modelo explica un $51 \%$ de la varianza total, por tanto, se cumple el requisito mínimo (>50\%), presenta una adecuación muestral de un $\mathrm{KMO}=.892$ y un alfa de Cronbach de .900 , estadísticamente satisfactorio (Lloret-Segura et al., 2014).

El análisis permitió establecer dos dimensiones relacionadas con la Satisfacción del alumnado (F1) y Rol Docente (F2). La primera explica el $41 \%$ de la varianza y el segundo factor el $10 \%$ restante. Es bueno precisar que se eliminó una de las variables (V8) porque cargaba de forma casi igualitaria en las dos dimensiones. En definitiva, los dos factores quedaron compuestos de la siguiente forma (ver tabla 2), y se corresponden con los siguientes enunciados.

Factor 1 (F1). Satisfacción: Tiene que ver con el grado de satisfacción que mostraron los participantes (alumnado) tras finalizar el proyecto. Está compuesto por las siguientes categorías: ha sido aburrido, he perdido el tiempo, he aprendido menos cosas que otras veces, no ha sido interesante, ya no me interesa el tema, ha sido inútil, no me ha gustado la forma de trabajar, y el profesor te ha ayudado en las tareas.

Factor 2 (F2). Rol Docente: Se refiere a la función desempeñada por el docente desde el inicio hasta finalizar el proyecto. La realización de dichas funciones promovió ciertas actitudes y sensaciones que el alumnado valora en los siguientes ítems. He leído poco, no he comprendido lo que hemos hecho, me he distraído, el docente no ha dado las instrucciones claras, se han compartido materiales entre los compañeros; ahora resulta más difícil relacionarme con los compañeros, trabajando en grupo no se ha conseguido hacer bien la tarea, el tamaño del grupo no ha sido el adecuado, no he estado a gusto con mis compañeros; el profesor ha hecho un seguimiento del trabajo, el profesor ha indicado la calidad del trabajo presentado. 
Tabla 2. Matriz de componentes rotados

\begin{tabular}{|c|c|c|c|}
\hline \multicolumn{2}{|c|}{ VARIABLES } & F1 & F2 \\
\hline V1 & Ha sido aburrido & $\mathbf{0 . 7 5 9}$ & 0.488 \\
\hline V2 & He perdido el tiempo & $\mathbf{0 . 7 0 4}$ & 0.506 \\
\hline V3 & He aprendido menos cosas que otras veces & $\mathbf{0 . 6 3 9}$ & 0.496 \\
\hline V4 & He leído poco & 0.260 & $\mathbf{0 . 4 5 8}$ \\
\hline V5 & Ha sido interesante & $\mathbf{0 . 7 6 1}$ & 0.539 \\
\hline V6 & Me he distraído & 0.443 & $\mathbf{0 . 6 9 1}$ \\
\hline V7 & Ya no me interesa el tema & $\mathbf{0 . 5 6 2}$ & 0.495 \\
\hline V9 & Ha sido inútil & $\mathbf{0 . 7 8 3}$ & 0.423 \\
\hline V10 & No me ha gustado la forma de trabajar & $\mathbf{0 . 7 4 8}$ & 0.500 \\
\hline V11 & El profesor te ha ayudado en las tareas & 0.726 & 0.392 \\
\hline V12 & El docente ha dado instrucciones claras & 0.491 & $\mathbf{0 . 8 0 5}$ \\
\hline V13 & Han compartido materiales entre los compañeros & 0.151 & $\mathbf{0 . 4 7 4}$ \\
\hline V14 & Has estado a gusto con los compañeros & 0.433 & $\mathbf{0 . 5 2 3}$ \\
\hline V15 & Ahora resulta más difícil relacionarse con los compañeros & 0.418 & $\mathbf{0 . 5 2 8}$ \\
\hline V16 & Trabajando en grupo no han conseguido hacer bien la tarea & 0.427 & $\mathbf{0 . 7 3 4}$ \\
\hline V17 & El tamaño del grupo no ha sido adecuado & 0.513 & $\mathbf{0 . 6 6 3}$ \\
\hline V18 & El profesor ha hecho un seguimiento del trabajo & 0.556 & $\mathbf{0 . 7 1 8}$ \\
\hline V19 & El profesor ha indicado la calidad del trabajo presentado & 0.487 & $\mathbf{0 . 7 0 6}$ \\
\hline V20 & & & \\
\hline
\end{tabular}

Fuente. Análisis realizado con el programa estadístico Unrestricted Factor Analysis, a partir de los estudios de casos llevados a cabo en el Instituto Fabio Amable Mota, septiembre-diciembre de 2017.

\section{RESULTADOS}

La evaluación de los proyectos se distribuye en varias categorías que se muestran a continuación. Estas son: los roles de profesores y alumnos, la organización de espacios y tiempos, la calidad de las tareas y adecuación de los recursos digitales y la valoración del proyecto por profesores, alumnos y evaluador. Todos estos elementos se analizan con el fin evaluar la satisfacción del alumnado y profesorado con CSCL. 


\subsection{ROLES DE PROFESORES Y ALUMNOS}

Refiriéndonos a los roles de alumnado se apreció que cumplían sus tareas de manera responsable, cooperaban con sus compañeros, y cada cual sabía el rol que le correspondía. Se considera que esto es debido a que dentro de los grupos se crearon roles (control de calidad, dinamizador, coordinador...) y, se distribuyeron las fases del trabajo. La distribución facilitó la comunicación, la interactividad y el liderazgo. Sin embargo, aunque se les vio muy unidos, la colaboración con integrantes de otros grupos era algo menor (caso B).

Por el contrario, la participación extra-grupal en el caso A fue algo mayor que en el caso B debido a que la docente fomentó la cooperación y colaboración entre los grupos. Por ejemplo, todos los grupos socializaron el tema e intercambiaron las estrategias seguidas para la realización del proyecto. No obstante, en líneas generales los roles de docentes y alumnos fueron bastante buenos (ver fig. 1).

Figura 1. Triangulación de datos sobre los roles de docentes y alumnos
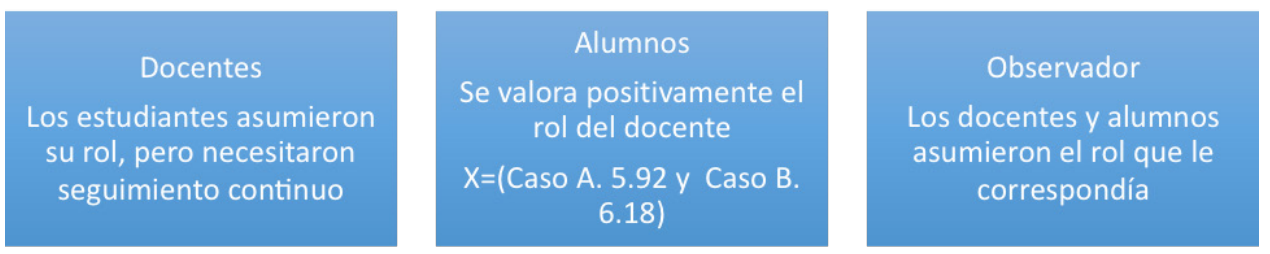

Fuente. Las fichas del observador, fichas del profesorado y rúbrica cumplimentada por los alumnos.

Elaboración propia.

Si nos fijamos en la figura 1 el observador coincide con alumnos y docentes en el buen desempeño de ambos, especialmente del profesor. Su rol fue determinante en el éxito de la tarea, interviniendo para resolver conflictos, y dar seguimiento de las tareas dentro y fuera del aula (plataforma Edmodo) para tener una evaluación justa de las mismas.

\subsection{ORGANIZACIÓN DE ESPACIOS Y TIEMPOS}

La organización del tiempo y espacio fue correcta, tomando en consideración la cantidad de alumnos, recursos disponibles y que, previo al desarrollo del tema, los alumnos requerían familiarizarse con el vocabulario sobre el tema de la contaminación en ingles, ya que no tenían pleno dominio de este. Por tanto, se adecuó la temporización para estudiar este contenido. Las aulas eran amplias, con mucha luminosidad, aunque con respecto al caso B eran pequeñas considerando cantidad de alumnos (32).

\subsection{CALIDAD DE LAS TAREAS Y ADECUACIÓN DE LOS RECURSOS DIGITALES}

Las tareas que desarrollaron los alumnos fueron relevantes y los criterios para abordarlas fueron adecuados. 
Tabla 3. Comparación de la planificación de los casos

\begin{tabular}{|c|c|c|}
\hline & $\begin{array}{c}\text { Caso A. } \\
\text { Tercero de Informática }\end{array}$ & $\begin{array}{c}\text { Caso B. } \\
\text { Cuarto de Informática }\end{array}$ \\
\hline Objetivos & \multicolumn{2}{|c|}{$\begin{array}{l}\text {-Investigar las causas y las consecuencias de la acumulación de los desechos sólidos, } \\
\text { su consecuente contaminación del ambiente escolar, con el fin de proponer soluciones } \\
\text { alternativas a la problemática. } \\
\text {-Motivar un pensamiento reflexivo y crítico sobre el tema medioambiental en toda la } \\
\text { comunidad educativa, con líneas de solución concretas. }\end{array}$} \\
\hline Estrategias & $\begin{array}{l}\text {-Aprendizaje Basado en la Resolución de } \\
\text { Problemas. } \\
\text {-lista de preguntas relacionadas con el problema } \\
\text {-Organizar charlas, paneles con la junta de vecinos } \\
\text {-Aprendizaje colaborativo (grupos de } 4 \text { a } 5 \\
\text { integrantes al azar). } \\
\text {-Colaboración entre colegas } \\
\text {-Tutorías de grupo e individual } \\
\text {-Tutoría entre iguales } \\
\text {-Enseñanza recíproca (colaboración entre los } \\
\text { grupos } \\
\text {-Redes Sociales } \\
\text {-Debates virtuales } \\
\text {-Empleo de recursos TIC para entregar tareas y } \\
\text { diseñar el proyecto } \\
\text {-Clases magistrales }\end{array}$ & $\begin{array}{l}\text {-Aprendizaje Basado en la } \\
\text { Resolución de Problemas. } \\
\text {-lista de preguntas relacionadas con } \\
\text { el problema } \\
\text {-Organizar charlas, paneles con la } \\
\text { junta de vecinos } \\
\text {-Aprendizaje colaborativo (grupos de } \\
4 \text { a } 5 \text { integrantes por orden alfabético). } \\
\text {-Colaboración entre colegas } \\
\text {-Tutoría de grupo e individual } \\
\text {-Técnica del puzzle } \\
\text {-El flipped classroom o aula invertida } \\
\text {-Redes sociales } \\
\text {-Empleo de recursos TIC para } \\
\text { entregar tareas y diseñar el proyecto } \\
\text {-Clases magistrales }\end{array}$ \\
\hline Recursos & $\begin{array}{l}\text {-Edmodo } \\
\text {-Laboratorio de informática } \\
\text {-Equipos móviles } \\
\text {-Proyectores } \\
\text {-Paquete office } \\
\text {-Youtube } \\
\text {-Internet } \\
\text {-Papelería (carteles, murales...) } \\
\text {-Bolsas de basura } \\
\text {-Documentos digitales } \\
\text {-Guía de estudio }\end{array}$ & $\begin{array}{l}\text {-Edmodo } \\
\text {-Facebook } \\
\text {-Laboratorio de informática } \\
\text {-Equipos móviles } \\
\text {-Proyectores } \\
\text {-Paquete office } \\
\text {-Youtube } \\
\text {-Internet } \\
\text {-Papelería (carteles, murales...) } \\
\text {-Bolsas de basura } \\
\text {-Pizarra tradicional } \\
\text {-TV plasma } \\
\text {-Documentos digitales } \\
\text {-Guía de estudio }\end{array}$ \\
\hline Evaluación & $\begin{array}{l}\text { Iniciativa, capacidad de liderazgo, comunicación, } \\
\text { implicación en las tareas, tolerancia, creatividad, } \\
\text { calidad de las tareas, puntualidad de entrega, } \\
\text { cooperación y colaboración con otros } \\
\text { compañeros. (Diagnóstica, formativa, continua y } \\
\text { sumativa) }\end{array}$ & $\begin{array}{l}\text { Iniciativa, capacidad de liderazgo, } \\
\text { comunicación, implicación en las } \\
\text { tareas, tolerancia, creatividad, calidad } \\
\text { de las tareas, puntualidad de entrega } \\
\text { cooperación y colaboración con otros } \\
\text { compañeros (Diagnóstica, formativa, } \\
\text { continua y sumativa) }\end{array}$ \\
\hline
\end{tabular}

Fuente. Fichas del docente y observador. Elaboración propia. 
Estudios Pedagógicos XLVII N² 2: 79-97, 2021

SATISFACCIÓN DEL PROFESORADO Y ALUMNADO CON EL EMPLEO DE METODOLOGÍAS DE APRENDIZAJE COLABORATIVO MEDIADA POR LAS TIC: DOS ESTUDIOS DE CASOS

En cuanto a la planificación de la sección se apreció una planificación muy completa. Los objetivos, tiempos, actividades estaban bien definidos y distribuidos. Las tareas fueron explicadas con mucha claridad, paso a paso y siguiendo los lineamientos de la planificación. (ver tabla 3). La relevancia y coherencia de esta con los objetivos de aprendizaje se refleja en la anotación del observador.

La docente empezó presentando los objetivos al grupo, luego les indicó a los alumnos que utilizaran la técnica de mapeo para analizar la problemática planteada. Durante el desarrollo de la sección los alumnos trabajaron en grupo con las computadoras, empleando "enseñanza recíproca" para elaborar cuestionarios, indagar sobre el tema y preparar exposiciones (Observador Caso A).

El maestro ejecutó diversas estrategias de aprendizaje con los estudiantes para desarrollar cada actividad; como son, seguimiento de las tareas, técnica del puzzle, trabajo en equipo, redes sociales. Todas las actividades que se desarrollaron estaban muy relacionadas con los objetivos de aprendizaje, con lo cual las tareas eran muy relevantes (Observador Caso B).

Además, se observa una gran sincronía en los elementos de planificación elegidos para cada grupo. Donde se observa más disparidad es en las estrategias y los recursos. Por ejemplo, el docente del caso A apostó por la tutoría entre iguales que permite la adquisición de conocimientos y habilidades a través de la ayuda activa y el apoyo entre iguales o compañeros equivalentes (Topping, 2015). Mientras en el caso B se utilizó la técnica del puzzle. Aquí cada estudiante debía buscar un fragmento de información que se asigna previamente. La información obtenida se confronta con los demás integrantes del grupo y, a partir de la misma se construye un proyecto en común (Vargas-Cruz, 2017). Todo esto se realizó con el fin de crear interdependencia positiva entre compañeros.

En cuanto a la utilización de recursos TIC, se emplearon en el centro educativo y en el hogar. Además, docentes y observadores señalaron que fueron tan útiles como los materiales tradicionales y sirvieron para potenciar el rendimiento académico. No obstante, pese a la buena calidad de los recursos digitales del centro, la cantidad era escasa teniendo en cuenta el número de alumnos.

\subsection{VALORACIÓN GENERAL DE LOS CASOS (DOCENTE, EVALUADOR Y ALUMNADO)}

Las valoraciones que aquí se plasman se deprenden de la ficha docente, del observador y de la rubrica cumplimentada en el alumnado (diferencial semántico). Se analizan las valoraciones del docente y observador con respecto a la metodología, recursos, actividades, satisfacción, competencias adquiridas, limitaciones y los hallazgos que arrojó el diferencial semántico. Todos estos elementos de analizan con el fin de identificar los aspectos que han influido en la satisfacción de alumnado y docente.

El análisis de estudio de las fichas (docente y evaluador) nos permite afirmar que se han conseguido los objetivos propuestos; los alumnos trabajaron correctamente los contenidos y, según la concepción del docente. Además, en ambas anotaciones (profesor-observador), se aprecia que los contenidos le parecieron bastante interesante a los estudiantes. A excepción del "caso B", en donde alrededor de un 34\% del alumnado manifestó no estar tan 
interesado en el tema trabajado, ya que sus posturas estaban más cercanas al 1 (totalmente en desacuerdo) que al 7 (totalmente de acuerdo) (ver fig. 2).

Figura 2. Interés en los contenidos trabajados por los alumnos del Caso A y B

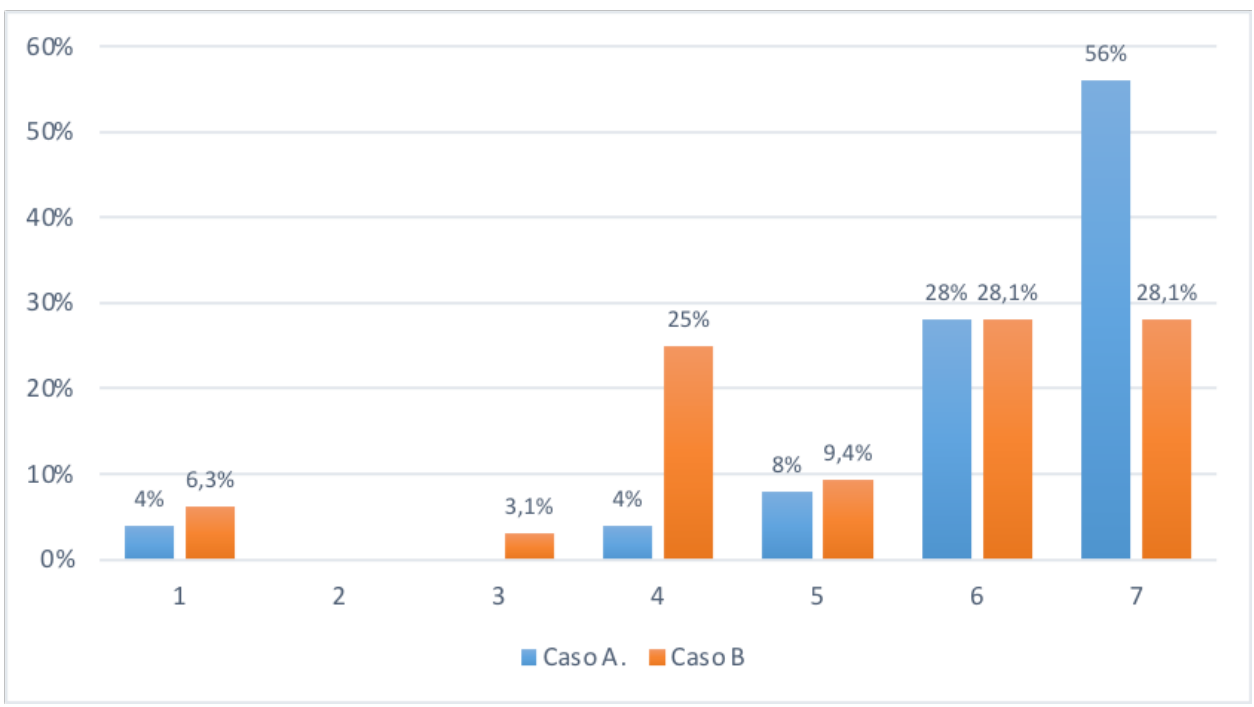

Fuente. Rúbrica cumplimentada por los alumnos. Elaboración propia.

De igual forma el maestro se mostró muy satisfecho con la metodología utilizada y los materiales didácticos. En ese sentido, el docente y observador expresaron que las actividades desarrolladas sirvieron para trabajar los contenidos, despertaron la curiosidad del alumnado, facilitaron el trabajo activo y colaborativo de los mismos y fomentaron su creatividad.

De acuerdo con las valoraciones del profesorado y del observador, el clima de clase de ambos grupos/grados era bueno, lo que propició un ambiente idóneo para desarrollar aprendizajes significativos, habilidades de trabajo en equipo, enseñanza reciproca y habilidades TIC.

La organización de espacios y tiempos han sido correctos para llevar a cabo las actividades planificadas, se ha trabajado la mayor parte del tiempo de clase con un grupo fijo que el docente formó al inició del proyecto, teniendo en cuenta que cada grupo fuera heterogéneo y con una cantidad de alumnos adecuada.

La evaluación del proyecto que incluye los dos casos en su conjunto ha sido muy buena, situándose la valoración del docente en bastante y mucho. En dicho proceso se promovieron actitudes positivas del alumnado hacia el aprendizaje y, en mayor medida de los alumnos hacia otros compañeros y hacia el profesor. Los docentes se mostraron muy satisfechos con lo realizado, no obstante, señalaron que los alumnos aventajados aprovecharon más las actividades que los alumnos con dificultades de aprendizaje, a los que les costó algo más conseguir los objetivos. Además, al principio de formación de los grupos hubo algunos problemas de adaptación, ya que algunos deseaban trabajar con otros compañeros (ver fig. 3). 
Figura 3. Limitaciones encontradas trabajando con CSCL

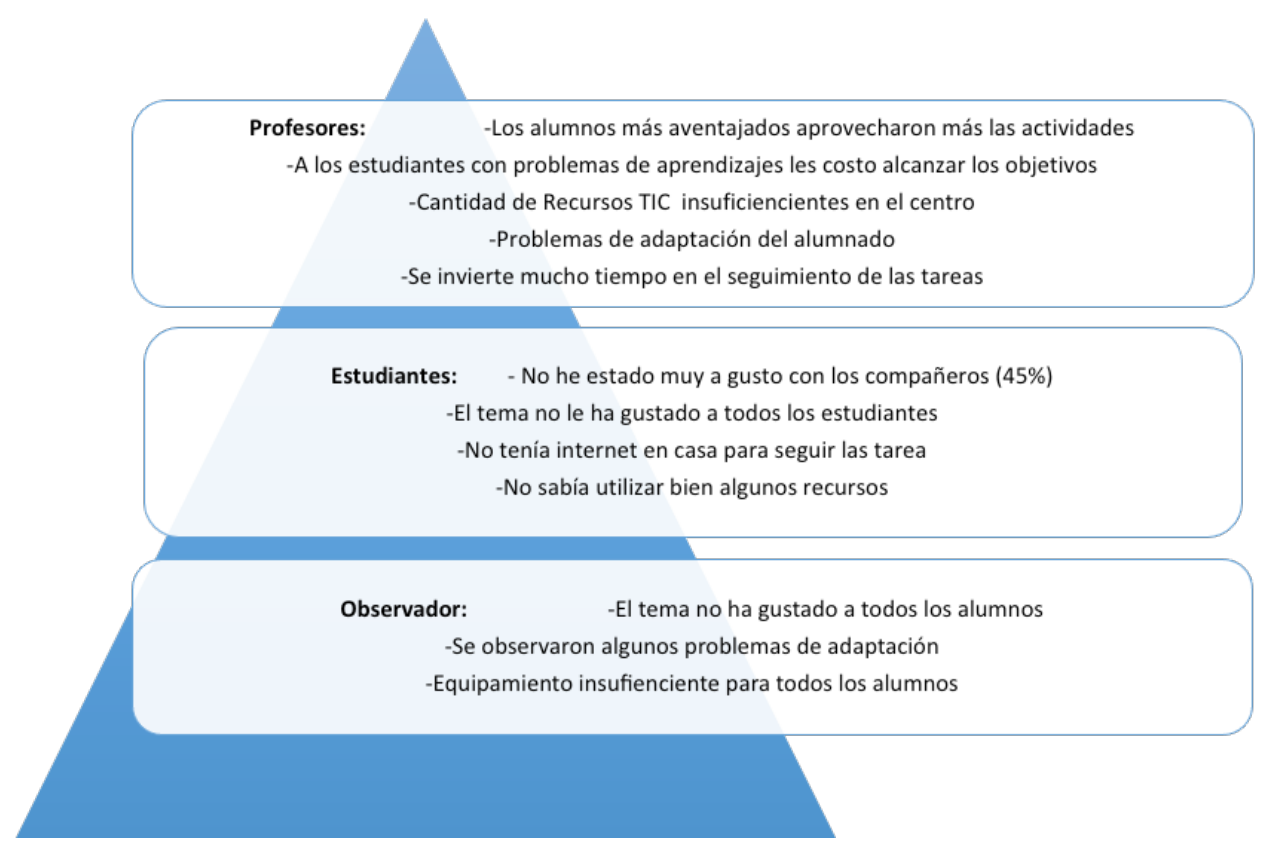

Fuente. Las fichas del observador, fichas del profesorado y rúbrica cumplimentada por los alumnos.

Elaboración propia.

Por otro lado, acorde con la media de la rúbrica analizada, los estudiantes que trabajaron con metodologías colaborativas con TIC valoran positivamente el uso de la misma para su enseñanza. Se puede apreciar que la media obtenida de ambos grupos se sitúa en 5.96 y 6.06 respectivamente, con una media general de 6.00. Las mismas se consideran sumamente alta tomando en consideración que la escala de los ítems iba del 1 al 7. En lo referente a la asimetría y curtosis se observa que el constructo está distribuido asimétricamente hacia la izquierda, con lo cual tiene un sesgo negativo, mientras que la curtosis tiene una distribución leptocurtica al ser $\mathrm{C}>0$. (ver tabla 4). 
Tabla 4. Valoración global del empleo de Metodologías Colaborativas Mediadas por las TIC por parte del alumnado

\begin{tabular}{|c|c|c|}
\hline Grados & \multicolumn{2}{|c|}{ Medidas de dispersión } \\
\hline \multirow{6}{*}{$\begin{array}{l}\text { 3ro. Informática } \\
\text { (Caso A) }\end{array}$} & Tendencias centrales & 25 \\
\hline & Media (X) & 6.06 \\
\hline & Mediana & 6.20 \\
\hline & Desviación Típica & .548 \\
\hline & Asimetría & -.336 \\
\hline & Curtosis & -.592 \\
\hline \multirow{6}{*}{$\begin{array}{l}\text { 4to. Informática } \\
\text { (Caso B) }\end{array}$} & & 32 \\
\hline & Media (X) & 5.96 \\
\hline & Mediana & 6.05 \\
\hline & Desviación Típica & .764 \\
\hline & Asimetría & -.847 \\
\hline & Curtosis & .823 \\
\hline \multirow{5}{*}{$\begin{array}{l}\text { Valoración Global } \\
\text { de los casos }\end{array}$} & Media (X) & 6.00 \\
\hline & Mediana & 6.10 \\
\hline & Desviación Típica & .674 \\
\hline & Asimetría & -.806 \\
\hline & Curtosis & .946 \\
\hline Sig. & & .847 \\
\hline
\end{tabular}

Fuente. Los estudios de casos llevados a cabo en el Instituto Fabio Amable Mota, septiembre-diciembre de 2017.

Así mismo se comprobó si existía alguna diferencia entre la valoración global de los grupos, pero al ser p>.05 se comprueba que los grupos son homogéneos en cuanto a su consenso sobre el empleo de metodologías colaborativas mediadas por las TIC para su proceso de enseñanza aprendizaje.

Según las dimensiones creadas que citamos previamente "Satisfacción y Rol docente" la percepción del alumnado es muy buena. Si nos fijamos en la media de estos grupos oscila entre 5.54 y 6.33 y solo en uno de los casos la desviación típica es superior a uno. Con lo cual existe consenso en la valoración del alumnado (ver tabla 5). 
Estudios Pedagógicos XLVII N² 2: 79-97, 2021

SATISFACCIÓN DEL PROFESORADO Y ALUMNADO CON EL EMPLEO DE METODOLOGÍAS DE APRENDIZAJE COLABORATIVO MEDIADA POR LAS TIC: DOS ESTUDIOS DE CASOS

Tabla 5. Valoración del alumnado sobre las metodologías colaborativas por dimensiones y grupos

\begin{tabular}{|c|c|c|c|}
\hline \multirow{2}{*}{$\begin{array}{c}\text { 3ro. Informática } \\
(\text { Caso A) }\end{array}$} & Dimensiones & Media & Desviación Típica \\
\cline { 2 - 4 } & F1. Satisfacción & 6.33 & .617 \\
\cline { 2 - 4 } & F2. Rol Docente & 5.92 & .623 \\
\hline \multirow{2}{*}{$\begin{array}{c}\text { 4to. Informática } \\
\text { (Caso B) }\end{array}$} & F1. Satisfacción & 5.54 & $\mathbf{1 . 1 2}$ \\
\cline { 2 - 4 } & F2. Rol Docente & 6.18 & .609 \\
\hline
\end{tabular}

Fuente. Los estudios de casos llevados a cabo en el Instituto Fabio Amable Mota, septiembre-diciembre de 2017.

Tras categorizar las dimensiones satisfacción y rol docente en (muy malo, malo, regular, bueno y muy bueno) se verificó si existían correlaciones entre dichos elementos según el grado. Para dicho análisis se utilizó el coeficiente de correlación de Spearman, ya que dichas variables no tenían una distribución normal. Basándonos en los niveles críticos obtenidos se puede afirmar que la variable satisfacción correlaciona significativamente con el grado $(\mathrm{p}<.001)$ y rol docente $(\mathrm{p}=.045)$. En la primera la correlación afecta negativamente y en la segunda de forma positiva (ver tabla 6).

Tabla 6. Análisis de correlación Spearman según el grado

\begin{tabular}{|c|c|c|c|c|}
\hline & & Grado & F1. satisfacción & F2. rol docente \\
\hline \multirow{2}{*}{ Grado } & Coeficiente de Correlación & 1 & $-.389 * *$ & .194 \\
\cline { 2 - 5 } & Sig. (bilateral) & - & $\mathbf{p}<.001$ & .149 \\
\hline \multirow{2}{*}{$\begin{array}{c}\text { F1. } \\
\text { Satisfacción }\end{array}$} & Coeficiente de Correlación & $-.389^{* *}$ & 1 & $.513^{*}$ \\
\cline { 2 - 5 } & Sig. (bilateral) & $\mathbf{p}<. \mathbf{0 0 1}$ & - & $\mathbf{. 0 4 5}$ \\
\hline \multirow{2}{*}{$\begin{array}{c}\text { F2. Rol } \\
\text { docente }\end{array}$} & Coeficiente de Correlación & .194 & $.513 *$ & 1 \\
\cline { 2 - 5 } & Sig. (bilateral) & 0,149 & $\mathbf{. 0 4 5}$ & - \\
\hline
\end{tabular}

Fuente. Los estudios de casos llevados a cabo en el Instituto Fabio Amable Mota, septiembre-diciembre de 2017.

\section{DISCUSIÓN Y CONCLUSIONES}

Los estudios de caso expuestos confirman el potencial del aprendizaje colaborativo mediado por las TIC para el proceso de enseñanza-aprendizaje y el importante papel de la orientación docente y la instrucción clara por parte del mismo. Dado que el profesor debe estar presente en todo momento para facilitar los andamiajes de aprendizaje articulando correctamente las actividades con las necesidades de los alumnos. Así mismo cobra gran relevancia la necesidad de coherencia entre los objetivos, el diseño pedagógico y el contexto de los alumnos (Hernández et al., 2014; Hernando, 2015; Foldnes, 2016). 
Desde el punto de vista de los objetivos planteados en este estudio se encontraron los siguientes resultados. En primer lugar, tras evaluar el grado de satisfacción del profesorado y alumnado cuando trabajan con las metodologías de aprendizaje colaborativo mediadas por las TIC en el proceso de enseñanza-aprendizaje, los hallazgos muestran un alto nivel de satisfacción, tanto por parte del alumnado $(X=6.00)$ como del profesorado.

En el caso de los docentes este alto nivel de satisfacción estuvo motivado sobre todo por la gran participación de los grupos en las actividades. Por su parte, el alumnado señaló que el empleo de estas metodologías fue una experiencia motivadora que mejoró sus habilidades sociales. No obstante, aunque la media global de ambos casos ( $X=5.96 ; 6.06)$ indican un alto grado de satisfacción del alumnado, se encontraron diferencias significativas en función del grado $(\mathrm{p}=<.001)$ y el rol que desempeñó el docente $(\mathrm{p}=.045)$. El grupo A se sintió más satisfecho que el grupo B al trabajar con metodologías colaborativas mediadas por las TIC. Probablemente el grupo A empatizó más con la temática objeto de estudio que el grupo B.

Aquí cobra vital importancia el hecho de elegir un eje temático de interés para la mayor parte del grupo con el fin de que estos se sientan atraídos y no pierdan la motivación. Sin embargo, hemos de señalar que será difícil llegar a un consenso para encontrar un tema idóneo; lo más recomendable sería crear una dinámica para despertar la curiosidad del alumnado, junto a la elección de un material didáctico ameno y práctico, que facilite la comprensión del contenido y que se adapte al contexto. Todo ello, dentro de un marco de ecosistemas tecnológicos colaborativos (García-Peñalvo, 2018). A esto se ha de añadir la concepción docente sobre el aprendizaje colaborativo asistido por computadores, puesto que es determinante para entender que el diseño de dichos entornos no se ha de limitar a una visión instrumental "centrada en los artefactos" sino que ha de complementarse con otras ciencias como la psicología, sociología, didáctica, etc., para atender a la demanda de los estudiantes, modificar sus comportamientos en la dirección de los sentimientos "positivos y motivadores" y mejorar su aprendizaje (Acosta et al., 2019; Williamson, 2017).

Además, según Noguera et al. (2018), este descontento también puede ser producto de experiencias anteriores insatisfactorias con actividades de aprendizaje colaborativo, las cuales influyen en la predisposición de los estudiantes hacia actividades de aprendizaje en equipo. De hecho, en uno de los casos el/la docente comentó en la ficha de observaciones "que uno de los alumnos quería realizar su trabajo individual y no se quería relacionar con los demás".

En cuanto al segundo objetivo comprobar la correlación entre el rol docente y la satisfacción del alumnado según el grado o nivel educativo, los hallazgos indicaron que el grado $(\mathrm{p}<.001)$ y el rol docente $(\mathrm{p}=.045)$ correlacionan significativamente con la motivación. En ese sentido, se comprobó que el alumnado más satisfecho con el empleo de CSCL es aquel que valora positivamente el rol asumido por el docente en el desarrollo de estas metodologías. Y es que como apuntan García-Valcárcel y Tejedor (2018); Rahimi y Karkami, (2015), el rol docente juega un papel crucial para motivar a los alumnos y ayudarles a alcanzar los objetivos.

En tercer lugar, con respecto a identificar las fortalezas y debilidades con que se encuentran los alumnos y profesores cuando emplean estas experiencias colaborativas; se ha de señalar, por una parte, que tanto docentes, como alumnado y evaluador señalaron como fortalezas las competencias adquiridas con respecto a las habilidades de comunicación, incremento de la motivación, desarrollo de la creatividad, conexión síncrona y asíncrona, 
Estudios Pedagógicos XLVII N² 2: 79-97, 2021

SATISFACCIÓN DEL PROFESORADO Y ALUMNADO CON EL EMPLEO DE METODOLOGÍAS DE APRENDIZAJE COLABORATIVO MEDIADA POR LAS TIC: DOS ESTUDIOS DE CASOS

mejora del aprendizaje, mejora del clima del aula; mayor autonomía, adquisición de competencias digitales, entre otras. Podemos decir entonces que esta metodología favorece la convivencia e integración social del alumnado, a la vez que potencia el desarrollo de responsabilidades individuales y colectivas que optimizan el aprendizaje (Cifuentes y Meseguer, 2015; Johnson y Johnson, 2009; Koschmann et al., 1996).

En contraposición, se encontraron problemas relacionados con aspectos técnicos, equipamiento insuficiente, problemas cognoscitivos en el manejo de equipos y aplicaciones, adaptación al inicio de los grupos. Además, en el momento de evaluar el trabajo los docentes manifestaron que les tomó mucho tiempo monitorizar todos los grupos en las fases presenciales, y verificar la participación e implicación de estos en las actividades, con el fin de no cometer injusticias.

Pese a la formación recibida previamente sobre metodologías de aprendizaje colaborativo con TIC, se comprueba que la evaluación sigue siendo uno de los aspectos donde el profesorado tiene más dificultades y es, por tanto, una problemática que merece más atención. El docente debe ser capaz de orientar al alumnado y realizar un seguimiento continuo que garantice una evaluación justa y no le reste tanto tiempo; en consecuencia, ha de considerar las TIC como un buen aliado la cual le apoya en esa labor de seguimiento y control (García, 2014; Gikandi, 2020; Thurston et al., 2007).

Las ventajas y desventajas aquí citadas concuerdan con estudios realizados previamente (Acosta et al., 2020; García-Merino et al., 2016; García-Valcárcel, 2015; Gikandi, 2020; Hernando, 2015; Rodríguez et al., 2017; Tamargo y Rodríguez, 2015), en donde se muestra que el empleo de CSCL no es la "panacea." Incluso, en ocasiones se generan expectativas que no se cumplen y causan gran desconcierto. Esto no quiere decir que dicha metodología no sea eficaz, sino todo lo contrario, pero requiere formación, constancia y, sobre todo implica cuestionarse continuamente "qué, por qué y cómo" funciona mi diseño pedagógico para aplicarlo en el aula.

Con relación al cuarto y último objetivo analizar las valoraciones del docente, alumnado y observador sobre los contenidos, metodologías, tiempo, agrupamiento, proceso de evaluación del proyecto, diferencias y similitudes; tras comparar ambos casos se observó coherencia entre los contenidos y actividades, adecuación en la planificación didáctica del docente. Así mismo, se pudo constatar que la colaboración existente entre los profesores enriqueció las estrategias y recursos utilizados. Con esto se comprueba lo expuesto por Johnson y Johnson (2009), quienes sostienen que cuando se toman decisiones previas, reflexionando sobre un buen diseño, articulación de objetivos con los contenidos, temporalización, etc., los resultados de aprendizaje son óptimos. También se comprobó que cuando el docente es formado previamente en CSCL su satisfacción es positiva y, por ende, su actitud (Kumar et al., 2010; Rubia y Guitert, 2014). En lo referente a las diferencias más destacables entre ambos grupos, se observó que se situaban en consideración al tema desarrollado. Por ejemplo, en el "caso B" alrededor de un 34\% del alumnado manifestó no estar tan interesado en el tema trabajado, mientras que el "caso A" solo un $8 \%$ declaró esta cuestión.

Los resultados expuestos no pueden ser generalizables, puesto que se trata de un estudio de casos y la población que enmarca no tiene un amplio rango de representatividad, ya que pertenecen a un colectivo de un solo centro educativo. No obstante, los hallazgos encontrados pueden servir de punto de partida para cualquier otra investigación que aborde nuevos modelos de enseñanza que están cambiando la forma de enseñar y aprender o, 
incluso de referencia para que las administraciones educativas fomenten la formación docente en CSCL. Se cree que, si el profesorado fuese concienciado sobre el impacto que produce este modelo de enseñanza, se formara adecuadamente en dichas metodologías y se le diese un seguimiento continuo; entonces se podría experimentar un incremento en los niveles de aprendizaje del alumnado y en las competencias claves (Jiménez et al., 2018).

Finalmente, consideramos que para incentivar la puesta en marcha de metodologías colaborativas mediadas por las TIC como las que se describen anteriormente. Es necesario dar más participación y autonomía a las escuelas en la toma de decisiones curriculares para que estas puedan plantear sus necesidades y, a partir de las mismas, desarrollar sus proyectos de centro (Hernando, 2015; Cruz, 2016).

\section{REFERENCIAS BIBLIOGRÁFICAS}

Acosta, R., Joo, J., Martín, A. V. y Hernández, A. (2020). Perception of Teachers on Collaborative Tools Knowledge Level Mediated by ICT and Their Experience with Students. International Journal Of Emerging Technologies In Learning (IJET), 15(11), 137-161. http://dx.doi. org/10.3991/ijet.v15i11.13121

Acosta, R., Martín, A. V. y Hernández, A. (2019). Uso de las Metodologías de Aprendizaje Colaborativo con TIC: Un análisis desde las creencias del profesorado. Digital Education Review, (35), 309-323. https://doi.org/10.1344/der.2019.35.309-323

Almerich, G., Suárez-Rodríguez, J., Díaz-García, I. y Orellana, N. (2019). Estructura de las competencias del siglo XXI en alumnado del ámbito educativo. Factores personales influyentes. Educación XX1, 23(1), 45-74. https://doi.org/10.5944/educxx1.23853

Auld, E., Rappleye, J. y Morris, P. (2019). PISA for Development: how the OECD and World Bank shaped education governance post-2015. Comparative Education, 55(2), 197-219. https://doi. org/10.1080/03050068.2018.1538635

Assiter, A. (2017). Transferable skills in higher education. Routledge.

Brannen, J. (2017). Mixing methods: Qualitative and quantitative research. Routledge.

Cañón, R., Grande de Prado, M. y Cantón Mayo, I. (2016). Brecha digital: impacto en el desarrollo social y personal. Factores asociados. Tendencias pedagógicas, 28, 115-132. https://doi. org/10.15366/tp2016.28.009

Cifuentes, P. y Meseguer, P. (2015). Trabajo en equipo frente a trabajo individual: ventajas del aprendizaje cooperativo en el aula de traducción. Tonos Digital, 28(0). http://www.tonosdigital. es/ojs/index.php/tonos/article/viewArticle/1213

Cruz, S. M. (2016). Autonomía de centros: del papel a las aulas. InForum Aragón, 12-16. http://feae. eu/wp-content/uploads/2014/05/Forum-Aragón-17.pdf\#page=12

Esteve, F. (2009). Bolonia y las TIC: de la docencia 1.0 al aprendizaje 2.0. La cuestión universitaria, 5, 58-67. http://polired.upm.es/index.php/lacuestionuniversitaria/article/view/3337

Foldnes, N. (2016). The flipped classroom and cooperative learning: Evidence from a randomised experiment. Active Learning in Higher Education, 17(1), 39-49. https://doi. org/10.1177/1469787415616726

García, L. M. (2014). Evaluación formativa de los aprendizajes en el contexto universitario: Resistencias y paradojas del profesorado. Educación XX1, 17(2). https://doi.org/10.5944/ educxx1.17.2.11478

García-Merino, J. D., Urionabarrenetxea, S. y Bañales-Mallo, A. (2016). Cambios en metodologías docentes y de evaluación: ¿Mejoran el rendimiento del alumnado universitario? Revista electrónica de investigación educativa, 18(3), 1-18. https://redie.uabc.mx/redie/article/ view/691 
Estudios Pedagógicos XLVII N² 2: 79-97, 2021

SATISFACCIÓN DEL PROFESORADO Y ALUMNADO CON EL EMPLEO DE METODOLOGÍAS DE APRENDIZAJE COLABORATIVO MEDIADA POR LAS TIC: DOS ESTUDIOS DE CASOS

García-Peñalvo, F. J. (2018). Ecosistemas tecnológicos universitarios. En J. Gómez (Ed.), Análisis de las TIC en las Universidades Españolas (pp. 164-170). CRUE: Universidades Españolas.

García-Valcárcel, A. (2015). Proyectos de trabajo colaborativo con TIC. Síntesis.

García-Valcárcel, A. y Tejedor, F. J. (2018). Valoración del trabajo colaborativo en los procesos de enseñanza-aprendizaje en entornos escolares con alto nivel TIC. Estudios sobre Educación, (34), 155-175. https://doi.org/10.15581/004.34.155-175

Gikandi, J.W. (2020). Towards a theory of formative assessment in online higher education. En Learning and Performance Assessment: Concepts, Methodologies, Tools, and Applications (pp. 389-413). IGI Global.

Hernández, N., González, M. y Muñoz, P. (2014). La planificación del aprendizaje colaborativo en entornos virtuales. Comunicar, 42, 25-33. https://doi.org/10.3916/C42-2014-02

Hernando, A. (2015). Viaje a la escuela del siglo XXI: así trabajan los colegios más innovadores del mundo. Fundación Telefónica.

Imbernon, F. M. y Medina, J. L. (2008). Metodología participativa en el aula universitaria. La participación del alumnado. Octaedro.

Jiménez, D., González, J. y Tornel, M. (2018). Formación del profesorado universitario en metodologías y su incidencia en el aula. Estudios Pedagógicos, 44(3), 157-172. http://dx.doi. org/10.4067/S0718-07052018000300157

Johnson, D. W. y Johnson, R. T. (2009). An educational psychology success story: Social interdependence theory and cooperative learning. Educational researcher, 38(5), 365-379. https://doi.org/10.3102/0013189X09339057

Koschmann, T., Kelson, A. C., Feltovich, P. J. y Barrows, H. S. (1996). Computer-supported problembased learning: A principled approach to the use of computers in collaborative learning. En T. Koschmann (Ed.), CSCL: Theory and practice of an emerging paradigm, (pp. 83-124). Routledge.

Kumar, V., Gress, C., Hadwin, A. y Winne, P. H. (2010) Assessing Process in CSCL: An Ontological Approach. International Journal of Computers in Human Behavior, 26(5), 825-834. https://doi. org/10.1016/j.chb.2007.07.004

Lugo, M. y Ithurburu, V. (2019). Políticas digitales en América Latina. Tecnologías para fortalecer la educación de calidad. Revista Iberoamericana De Educación, 79(1), 11-31. https://doi.org/ https://doi.org/10.35362/rie7913398

Lloret-Segura, S., Ferreres-Traver, A., Hernández-Baeza, A. y Tomás-Marco, I. (2014). El Análisis Factorial Exploratorio de los Ítems: una guía práctica, revisada y actualizada. Anales de Psicología, 30(3), 1151-1169. https://doi.org/10.6018/analesps.30.3.199361

Ministerio de Educación de República Dominicana, MINERD. (2017). Diseño Curricular Nivel Secundario: segundo ciclo 4to, 5to y 6to, versión preliminar. Minerd.

Noguera, I., Guerrero-Roldán, A. E. y Masó, R. (2018). Collaborative agile learning in online environments: Strategies for improving team regulation and project management. Computers \& Education, 116, 110-129. https://doi.org/10.1016/j.compedu.2017.09.008

Organización de las Naciones Unidas para la Educación, la Ciencia y la Cultura, UNESCO (2015). Educación 2030 Declaración de Incheon. Hacia una educación inclusiva, equitativa y de calidad y un aprendizaje a lo largo de la vida para todos. https://bit.ly/2IWJ8K8

Portillo-Torres, M. C. (2017). Educación por habilidades: Perspectivas y retos para el sistema educativo. Revista Educación, 41(2), 1-22. http://dx.doi.org/10.15517/revedu.v41i2.21719

Rahimi, M. y Karkami, F. H. (2015). The Role of Teachers' Classroom Discipline in Their Teaching Effectiveness and Students' Language Learning Motivation and Achievement: A Path Method. Iranian Journal of Language Teaching Research, 3(1), 57-82. https://files.eric.ed.gov/ fulltext/EJ1127336.pdf

Reis, R., Rodriguez, C., Lyra, K. y Isotani, S. (2017). Estrutura Ontológica para representar Papéis Colaborativos Afetivos em ambientes CSCL. Revista Brasileira de Informática na Educação, 25(02), 87. http://dx.doi.org/10.5753/rbie.2017.25.02.87 
Rodríguez, A. I., García-Riaza, B. y Gómez, M. C. (2017). Collaborative learning and mobile devices: An educational experience in Primary Education. Computers in Human Behavior, 72, 664-677. https://doi.org/10.1016/j.chb.2016.07.019

Rubia, B. y Guitert, M. (2014). ¿La revolución de la enseñanza? El aprendizaje colaborativo en entornos virtuales (CSCL). Comunicar, 21(42), 10-14. https://doi.org/10.3916/C42-2014-a2

Sangrà, A., Vlachopoulos, D. y Cabrera, N. (2012). Building an inclusive definition of e-learning: An approach to the conceptual framework. The International Review of Research in Open and Distributed Learning, 13(2), 145-159. https://doi.org/10.19173/irrodl.v13i2.1161

Schwanitz, D. (2018). La cultura: todo lo que hay que saber. Taurus.

Tamargo, P. M. y Rodríguez, C. (2015). Implicaciones del aprendizaje cooperativo en educación secundaria obligatoria. Revista de estudios e investigación en psicología y educación, 109-114. https://doi.org/10.17979/reipe.2015.0.01.547

Thurston, A., Van de Keere, K., Topping, K. J., Kosack, W., Gatt, S., Marchal, J., Mestdagh, N., Schmeinck, D., Sidor, W. y Donnert, K. (2007). Aprendizaje entre iguales en Ciencias Naturales de Educación Primaria: Perspectivas teóricas y sus implicaciones para la práctica en el aula. Electronic Journal of Research in Education Psychology, 5(13), 477-496. https://www. redalyc.org/articulo.oa?id=293121946002

Topping, K. (2015). Peer tutoring: old method, new developments/Tutoría entre iguales: método antiguo, nuevos avances. Infancia y aprendizaje, 38(1), 1-29. https://doi.org/10.1080/0210370 2.2014 .996407

Torrego, J.C. y Negro, A. (2012). Aprendizaje cooperativo en las aulas. Alianza Editorial.

Vaillant, D. y Zidán, E. R. (2018). Perspectivas de UNESCO y la OEI sobre la calidad de la educación. In H. Monarca (Coord.), Calidad de la Educación en Iberoamérica: Discursos, políticas y prácticas, (pp.136-154). Dykinson.

Van-Laar, E., Van-Deursen, A. J., Van-Dijk, J. A. y de Haan, J. (2017). The relation between 21stcentury skills and digital skills: A systematic literature review. Computers in human behavior, 72 , 577-588. https://doi.org/10.1016/j.chb.2017.03.010

Vargas-Cruz, J. (2017). Experiencia con la técnica puzzle de Aronson para contenidos de cálculo diferencial. Pensamiento y Acción, (22). https://revistas.uptc.edu.co/revistas/index.php/ pensamiento_accion/article/view/7401

Vega-Gil, L. (2016). Modelos globales de formación de profesores. La calidad basada en los procesos de transición. En J. M. Fernández Soria et al. (Eds.), La formación inicial del profesorado de educación secundaria (pp. 122-135). Tirant lo Blanch.

Williamson, B. (2017). Moulding student emotions through computational psychology: Affective learning technologies and algorithmic governance. Educational Media International, 54(4), 1-22. https://doi.org/10.1080/09523987.2017.1407080 
